\title{
Position Sensorless Control of BLDC Motors Based on Global Fast Terminal Sliding Mode Observer
}

\author{
Xiaoyuan Wang ${ }^{\dagger}$, Tao $\mathrm{Fu}^{*}$, and Xiaoguang Wang* \\ †, ${ }^{*}$ School of Electrical Engineering and Automation, Tianjin University, Tianjin, China
}

\begin{abstract}
The brushless DC motor (BLDCM) has many advantages. As a result, it is widely used in electric vehicle (EV) drive systems. To improve the reliability of the motor control system, a position sensorless control strategy based on a sliding mode observer (SMO) is proposed. The global fast terminal sliding mode observer (GFTSMO) is proposed to enhance the control performance of the SMO control system. The advantages of the linear sliding mode and the nonsingular terminal sliding mode (NTSM) are combined in the control strategy. The convergence speed of the system state is enhanced. The motor commutation point is obtained with the observation of the back EMF, and the instantaneous torque value of the motor is calculated. Therefore, the position sensorless control of the BLDCM is realized. Experimental results show that the proposed control strategy can improve the convergence speed, dynamic characteristics and robustness of the system.
\end{abstract}

Key words: Brushless DC motor (BLDCM), Electric vehicle (EV), Global fast terminal sliding mode observer (GFTSMO), Instantaneous torque control, Position sensorless control

\section{INTRODUCTION}

The brushless DC motor (BLDCM) has many advantages, such as high power density, high efficiency and good speed regulation performance. It is suitable for driving in electric vehicles (EV) [1]-[3]. The exact rotor position in conventional BLDCM commutation and regulation is obtained with a mechanical position sensor. However, the mechanical position sensor decreases the reliability and increases the cost and volume of the system. Therefore, the study of position sensorless control of BLDCMs has become a focus of academic research [4], [5].

Nowadays, there have been many research achievements in the study of the rotor position and the speed estimation. The methods include the back EMF method, the third harmonic EMF method and the observer method [6]-[8]. The detection circuits and the DC bus voltage midpoint are adopted to reconstruct the motor neutral point in the back EMF method. A filter circuit and a voltage comparison circuit are required. The back EMF zero-crossing point signal and commutation

Manuscript received Feb. 10, 2015; accepted Jun. 18, 2015

Recommended for publication by Associate Editor Dong-Hee Lee.

${ }^{\dagger}$ Corresponding Author: xywang62@tju.edu.cn

Tel: +86-13581882089, Tianjin University

*School of Electrical Engineering and Automation, Tianjin University, China tends to have a delay. The cost of the hardware increases while the system stability decreases. The third harmonic EMF method is only suitable for the motors with fixed winding inductance parameters, larger third harmonic component of the magnetic field and larger harmonic winding coefficient. A new system is constructed with the observer method. The inputs of the observer are measureable variables. The output signals are equivalent to the state of the original system under certain conditions. There are good characteristics of stability and robustness in the observer method. It has wide range applications. Observer methods include the full-order state observer, the Kalman filter observer and the sliding mode observer (SMO). Compared to the other observers, the SMO has low requirements of the accuracy of the mathematical model of the system. It has good robustness to system parameter perturbations and external disturbances [9]-[11].

The SMO can obtain the state of the uncertain object. The disturbance value of the object model can also be obtained with the SMO. The BLDCM control system will obtain good control performance if the SMO is applied. However, the chattering phenomenon will occur if using the conventional SMO [12]. The equivalent control cannot be directly obtained. An additional low-pass filter is required. An additional low-pass filter with adjustable frequency was designed according to the observation value in [13]. The chattering phenomenon was reduced. An adaptive filter based on the 
Kalman filter structure was designed in [14]. The continuous back EMF value was extracted from the switch signal. However, the phase delay of the observed value and the reduction of estimation accuracy were caused due to the filter. The usual solution is to compensate the phase delay of the equivalent control. However, it is difficult to compensate accurately. The effect on the phase delay of the estimated value cannot be eliminated completely.

The SMO include the linear sliding mode, the terminal sliding mode, the fast terminal sliding mode and the nonsingular terminal sliding mode (NTSM). The deviation between the system state and the given trajectory in linear sliding mode will asymptotic converge exponentially. It means that the system state will approach to the given trajectory continuously, but it will never reach the given trajectory. The terminal sliding mode control strategy was proposed in [15]. The nonlinear function was introduced to the sliding mode surface. The tracking error converged to the balance point in finite time. In [16], a continuous dynamic sliding mode control method was proposed for high-order mismatched disturbance attenuation in motion control systems using a high-order sliding mode differentiator. This method exhibits promising control performance in the presence of high-order matched and mismatched disturbances. The nonsingular terminal sliding mode controller was discussed in [17]. It was applied in robot control. A continuous nonsingular terminal sliding mode control approach was proposed for mismatched disturbance attenuation in [18]. The time taken to reach the desired set point from any initial state was guaranteed to be finite. A fast sliding surface was proposed in [19]. It converged faster to the equilibrium point, which drove the system state converge to the given trajectory in finite time. The control singular region is avoided directly in the design of the sliding mode control. In addition, the convergence property of terminal sliding mode in finite time is retained.

In view of the above problem, combining the advantages of the nonsingular terminal sliding mode and the high order sliding mode (HOSM), a BLDCM back EMF observer method based on a global fast terminal sliding mode observer (GFTSMO) is proposed in this paper. The nonsingular terminal sliding mode is designed to guarantee the system state convergence in finite time and global stability of the observer. The high order sliding mode control law of the observer is designed. The chattering of the SMO is eliminated effectively. The robustness of the observer is retained. Combined with the instantaneous torque control method, the torque ripple due to non-ideal back EMF and stator windings commutation was reduced. The effectiveness of the proposed method is verified by experimental results.

This paper is organized as follows. Back EMF estimation based on the conventional SMO is reviewed in Section II. To optimize the speed convergence performance, the global fast terminal sliding mode observer is presented in Section III.
Experimental results and analysis are illustrated in Section IV. Some conclusions are given in Section V.

\section{BACK EMF ESTIMATION BASED ON THE CONVENTIONAL SMO}

For a BLDCM with non-sinusoidal back EMF waveform, the voltage equation can be expressed as:

$$
\left\{\begin{array}{l}
L \frac{\mathrm{d} i_{a b}}{\mathrm{~d} t}=-R i_{a b}-e_{a b}+u_{a b} \\
L \frac{\mathrm{d} i_{b c}}{\mathrm{~d} t}=-R i_{b c}-e_{b c}+u_{b c} \\
L \frac{\mathrm{d} i_{c a}}{\mathrm{~d} t}=-R i_{c a}-e_{c a}+u_{c a}
\end{array}\right.
$$

The state equation of the BLDCM can be expressed as:

$$
\dot{\boldsymbol{i}}_{s}=\frac{1}{L}\left(-\mathbf{R} \boldsymbol{i}_{s}-\boldsymbol{\xi}+\boldsymbol{u}_{s}\right)
$$

Where $\boldsymbol{i}_{s}=\left[\boldsymbol{i}_{a b}, \boldsymbol{i}_{b c}, \boldsymbol{i}_{c a}\right]^{\mathrm{T}}$ is the stator current vector.

$\mathbf{R}=\left[\begin{array}{ccc}R & 0 & 0 \\ 0 & R & 0 \\ 0 & 0 & R\end{array}\right]$ is the stator winding resistance.

$\boldsymbol{\xi}=\left[e_{a b}, e_{b c}, e_{c a}\right]^{\mathrm{T}}$ is the stator back EMF vector.

$\boldsymbol{u}_{s}=\left[u_{a b}, u_{b c}, u_{c a}\right]^{\mathrm{T}}$ is the stator terminal voltage.

The electromagnetic torque can be expressed as $\left[20,21^{]}\right.$:

$$
T_{e}=\frac{e_{a} i_{a}+e_{b} i_{b}+e_{c} i_{c}}{\omega}=\frac{e_{x y} i_{x}}{\omega}
$$

Where $\omega$ is the motor rotor speed. $e_{a}, e_{b}, e_{c}$ is the motor back EMF; $i_{a}, i_{b}, i_{c}$ is the motor stator current; and $e_{x y}$ is the difference of the back EMF.

According to the state equation of the BLDCM in equation (2), the conventional SMO is constructed as follows:

$$
\dot{\hat{\boldsymbol{i}}}_{s}=\frac{1}{L}\left(-\mathbf{R} \hat{\boldsymbol{i}}_{s}+\boldsymbol{u}_{s}+\boldsymbol{u}\right)
$$

Where $\hat{\boldsymbol{i}}_{s}=\left[\hat{\boldsymbol{i}}_{a b}, \hat{\boldsymbol{i}}_{b c}, \hat{\boldsymbol{i}}_{c a}\right]^{\mathrm{T}}$ are the estimated stator current signals. $\quad \boldsymbol{u}=\left[u_{1}, u_{2}, u_{3}\right]^{\mathrm{T}}$ is the input vector of the observer.

Subtracting equation (2) from equation (4), the observer of the stator current error can be obtained:

$$
\dot{\tilde{\boldsymbol{i}}}=\frac{1}{L}\left(-\mathbf{R} \tilde{\boldsymbol{i}}_{s}+\boldsymbol{\xi}+\boldsymbol{u}\right)
$$

Where $\tilde{\boldsymbol{i}}_{s}=\left[\tilde{\boldsymbol{i}}_{a b}, \tilde{\boldsymbol{i}}_{b c}, \tilde{\boldsymbol{i}}_{c a}\right]^{\mathrm{T}}$ is stator current error vector. $\tilde{i}_{a b}=\hat{i}_{a b}-i_{a b} \quad, \quad \tilde{i}_{b c}=\hat{i}_{b c}-i_{b c}, \quad \tilde{i}_{c a}=\hat{i}_{c a}-i_{c a}$. $\boldsymbol{\xi}=\left[e_{a b}, e_{b c}, e_{c a}\right]^{\mathrm{T}}$ is the stator back EMF vector.

The SMO is designed according to the above observer. The stator current errors are state parameters. The sliding mode surface and control law are constructed. The stator current 


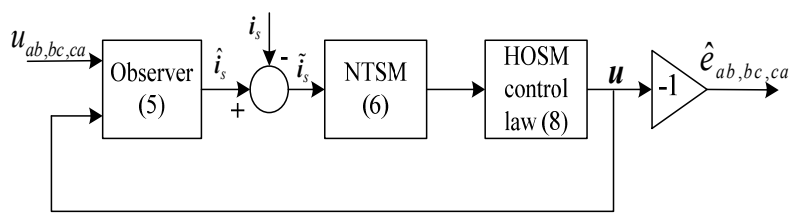

Fig. 1. Structure of nonsingular terminal SMO.

error moves along the sliding mode surface to the system equilibrium point with the effect of the sliding mode control, namely it converges to the zero point along the sliding mode surface. When the stator current error moves to the original point of the phase plane origin, $\tilde{\boldsymbol{i}}_{s}=\dot{\tilde{\boldsymbol{i}}}_{s}=0$. The value of the back EMF can be calculated according to the observer in equation (5). Therefore, the motor speed and commutation position information can be obtained. The estimation of the motor speed and position is achieved.

The nonsingular terminal SMO surface is designed as follows:

$$
\boldsymbol{s}=\tilde{\boldsymbol{i}}_{s}+\gamma \dot{\tilde{\boldsymbol{i}}}^{p / q}
$$

Where $\boldsymbol{s}=\left[s_{a b}, s_{b c}, s_{c a}\right]^{\mathrm{T}}, \boldsymbol{\gamma}=\left[\gamma_{a b}, \gamma_{b c}, \gamma_{c a}\right]^{\mathrm{T}}, \quad \gamma_{a b}>0$, $\gamma_{b c}>0, \quad \gamma_{c a}>0, p$ and $q$ are odd number, and $1<p / q<2$. $\dot{\tilde{\boldsymbol{i}}}_{S}{ }^{p / q}$ is defined as follows:

$$
\dot{\tilde{\boldsymbol{i}}}^{p / q}=\left[\dot{\tilde{\boldsymbol{i}}}_{a b}{ }^{p / q}, \dot{\tilde{\boldsymbol{i}}}_{b c}{ }^{p / q}, \dot{\tilde{i}}_{c a}{ }^{p / q}\right]^{\mathrm{T}}
$$

The control law is designed as follows:

$$
\boldsymbol{u}=\boldsymbol{u}_{e q}+\boldsymbol{u}_{n}
$$

with:

$$
\begin{gathered}
\boldsymbol{u}_{e q}=\mathbf{R} \tilde{\boldsymbol{i}}_{s} \\
\boldsymbol{u}_{n}=-\int_{0}^{t}\left[(\hat{L} q / p) \gamma^{-1 \dot{\hat{i}}_{s}^{2-p / q}}+\left(k^{\prime}+\eta\right) \operatorname{sgn}(s)+\mu s\right] d \tau
\end{gathered}
$$

Where $\operatorname{sgn}(s)=\left[\operatorname{sgn}\left(s_{a b}\right), \operatorname{sgn}\left(s_{b c}\right), \operatorname{sgn}\left(s_{c a}\right)\right]^{\mathrm{T}}, k^{\prime}>\|\dot{\xi}\|$, $\eta>0, \mu>0$.

When the system is in the high-order sliding mode, the dynamic system satisfies $\tilde{\boldsymbol{i}}_{s}=\dot{\tilde{\boldsymbol{i}}}_{s}=0$ according to equation (5). According to the equivalent control method [22], the motor back MEF is obtained:

$$
\hat{\boldsymbol{\xi}}=\left[\hat{e}_{a b}, \hat{e}_{b c}, \hat{e}_{c a}\right]^{\mathrm{T}}=-\boldsymbol{u}
$$

The motor speed is estimated with commutation signals. The period of the commutation cycle is the time of the motor rotation cycle. If the commutation period is $\Delta t$, the motor speed can be obtained $\omega=2 \pi / \Delta t$.

The structure of the nonsingular terminal SMO is shown in Fig. 1. Compared with the conventional linear SMO, the nonsingular terminal SMO can drive the control system converge to the desired trajectory in finite time. The convergence speed is fast when the system state is near the equilibrium point. However, when the system state is far away from the equilibrium point, the convergence speed becomes slower and the dynamic performance becomes worse [23]-[25].

\section{BACK EMF ESTIMATION BASED ON GFTSMO}

The convergence speed of the nonsingular terminal SMO is slower when the system state is far away from the equilibrium point. The convergence speed performance should be optimized to make the control system have better tracking accuracy and transient performance. A global fast terminal sliding mode observer is proposed in this paper. The sliding surface is designed as follows:

$$
\begin{gathered}
\boldsymbol{s}=\tilde{\boldsymbol{i}}_{s}+c \dot{\tilde{\boldsymbol{i}}}_{s}+\gamma \dot{\tilde{\boldsymbol{i}}}_{s}^{p / q} \\
\boldsymbol{\sigma}, \quad \begin{cases}\boldsymbol{\sigma} & |\dot{\boldsymbol{i}}|>1 \\
\boldsymbol{\sigma}|\dot{\tilde{\boldsymbol{i}}}|^{\zeta}, & |\dot{\tilde{\boldsymbol{i}}}| \leq 1\end{cases}
\end{gathered}
$$

The stator current error system can be made to converge to zero in finite time. If the SMO surface is designed as equation (12), the control law is designed as follows:

$$
\begin{gathered}
\boldsymbol{u}=\boldsymbol{u}_{e q}+\boldsymbol{u}_{n} \\
\boldsymbol{u}_{e q}=\mathbf{R} \tilde{\boldsymbol{i}}_{s} \\
\boldsymbol{u}_{n}=-\int_{0}^{t}\left[\frac{\dot{\tilde{\boldsymbol{i}}}_{s}}{c+p q^{-1} \gamma \operatorname{diag}\left(\dot{\tilde{\boldsymbol{i}}}_{s}^{(p / q)-1}\right)}+(k+h) \operatorname{sgn}(s)+\mu(s)\right] d \tau
\end{gathered}
$$

As shown in equation (13), the sliding mode surface of the GFTSMO consists of a linear sliding mode and a nonsingular terminal sliding mode. When the system state is far away from the equilibrium point $(|X|>1),|\dot{\tilde{i}}|>1, \sigma$ is a constant number larger than $1,1<p / q<2$, the linear sliding mode takes more effect than that of the nonsingular terminal sliding mode. As the convergence speed of the linear sliding mode is fast when it is far away from the equilibrium point, the system state convergence speed is improved. When the system state is near the equilibrium point $(|X| \leq 1),|\dot{\tilde{i}}| \leq 1, \zeta$ is a constant number larger than 1. The power function of the system state is introduced as the coefficient of the linear sliding mode. The coefficient decreases exponentially with the decrease of the state variable. The effect of the linear sliding mode function decreases gradually while the effect of the nonsingular terminal sliding mode increases. The convergence speed is enhanced when the system state is near the equilibrium point. The singularity problem is solved. Thus, the whole system has the advantages of global fast convergence. 


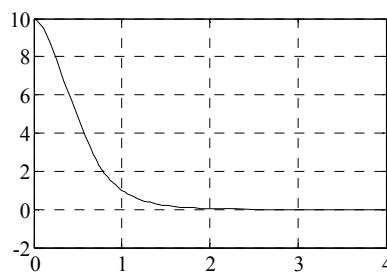

(a)

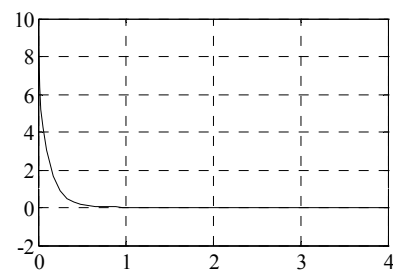

(b)
Fig. 2. The simulation of initial state $x=10$. (a) Conventional SMO. (b) The proposed SMO.

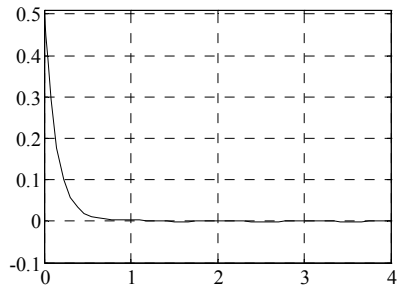

(a)

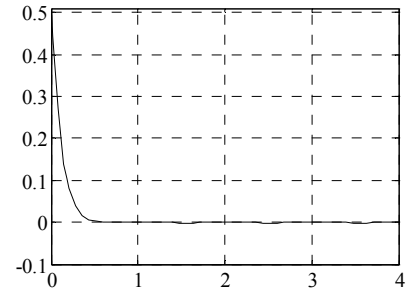

(b)
Fig. 3. The simulation of initial state $x=0.5$. (a) Conventional SMO. (b) The proposed SMO.

The convergence speed of the two kinds of SMO can be seen visually with the simulation comparison. Using a typical linear system is as an example, the state equation is as follows:

$J \ddot{x}=u(t)-d(t), d(t)=0.1 \sin (2 \pi t), J=1+0.5 \sin t$.

The initial states $x=10$ (far away from the equilibrium point) and $x=0.5$ (near the equilibrium point) were selected. Simulations were carried out under the above two different conditions. The performances with the conventional SMO and the GFTSMO were compared. The simulation results are shown as follows.

As can be seen from Fig. 2, the initial system state is far away from the equilibrium point. If the conventional SMO is used, it will take $1.9 \mathrm{~s}$ converge to the equilibrium point. If the proposed SMO is used, it will take $0.6 \mathrm{~s}$. It can be seen that the convergence speed is improved greatly. As shown in Fig. 3 , the initial state is near the equilibrium point. If the conventional SMO is used, the time converge to the equilibrium point is about $0.8 \mathrm{~s}$. When the proposed SMO is adopted, the time converge to the equilibrium point is $0.5 \mathrm{~s}$. The convergence characteristic of the proposed SMO is better than that of the conventional SMO.

In conclusion, compared with the conventional SMO, the proposed SMO can improve the convergence speed of the system state. Rapid global convergence is achieved.

To verify the stability of the proposed global fast terminal sliding mode control method, considering the following Lyapunov function:

$$
\boldsymbol{V}=\frac{1}{2} \boldsymbol{s}^{\mathrm{T}} \boldsymbol{s}
$$

Differentiate $\boldsymbol{V}$ :

$$
\begin{aligned}
& \dot{\boldsymbol{V}}=\boldsymbol{s}^{\mathrm{T}} \dot{\boldsymbol{s}}=\boldsymbol{s}^{\mathrm{T}}\left[\dot{\tilde{\boldsymbol{i}}}_{s}+\mathbf{C} \ddot{\tilde{\boldsymbol{i}}}_{s}+p q^{-1} \boldsymbol{\gamma} \operatorname{diag}\left(\dot{\tilde{\boldsymbol{i}}}_{s}^{(p / q)-1}\right) \ddot{\tilde{\boldsymbol{i}}}_{s}\right] \\
& =\boldsymbol{s}^{\mathrm{T}}\left[\dot{\tilde{\boldsymbol{i}}}_{s}+\left(\mathbf{C}+p q^{-1} \boldsymbol{\gamma} \operatorname{diag}\left(\dot{\tilde{\boldsymbol{i}}}_{s}^{(p / q)-1}\right)\right) \ddot{\tilde{\boldsymbol{i}}}_{s}\right] \\
& =\boldsymbol{s}^{\mathrm{T}}\left(\mathbf{C}+p q^{-1} \boldsymbol{\gamma} \operatorname{diag}\left(\dot{\tilde{\boldsymbol{i}}}_{s}^{(p / q)-1}\right)\right) \\
& {\left[\frac{\dot{\tilde{\boldsymbol{i}}}_{s}}{\mathbf{C}+p q^{-1} \boldsymbol{\gamma} \operatorname{diag}\left(\dot{\tilde{\boldsymbol{i}}}_{s}^{(p / q)-1}\right)}+\ddot{\tilde{\boldsymbol{i}}}_{s}\right]}
\end{aligned}
$$

According to (11) and (14)-(16), the following equation can be obtained:

$$
\begin{aligned}
& \dot{\tilde{\boldsymbol{i}}}_{s}=\frac{1}{L}\left(-R \tilde{\boldsymbol{i}}_{s}+\boldsymbol{\xi}+\boldsymbol{u}\right)=\frac{1}{L}\left(\boldsymbol{\xi}+\boldsymbol{u}_{n}\right) \\
& \ddot{\tilde{\boldsymbol{i}}}_{s}=\frac{1}{L}(\dot{\boldsymbol{\xi}}+\dot{\boldsymbol{u}})
\end{aligned}
$$

Therefore:

$$
\begin{aligned}
& \dot{V}=s^{\mathrm{T}}\left(c+p q^{-1} \gamma \operatorname{diag}\left(\dot{\tilde{i}}_{s}^{(p / q)-1}\right)\right) \\
& {\left[\frac{\dot{\tilde{i}_{s}}}{c+p q^{-1} \gamma \operatorname{diag}\left(\dot{\tilde{i}}_{s}^{(p / q)-1}\right)}+\ddot{\tilde{i}}_{s}+\frac{1}{L}(\dot{\xi}+\dot{u})\right]} \\
& =\frac{1}{L} s^{\mathrm{T}}\left(c+p q^{-1} \gamma \operatorname{diag}\left(\dot{\tilde{i}}_{s}^{(p / q)-1}\right)\right) \\
& {\left[\frac{L \dot{\tilde{i}}_{s}}{c+p q^{-1} \gamma \operatorname{diag}\left(\dot{\tilde{i}}_{s}^{(p / q)-1}\right)}+\ddot{\ddot{i_{s}}}+(\dot{\xi}+\dot{u})\right]} \\
& =\frac{1}{L} s^{\mathrm{T}}\left(c+p q^{-1} \gamma \operatorname{diag}\left(\dot{\tilde{i}}_{s}^{(p / q)-1}\right)\right) \\
& {[\dot{\xi}-(k+\eta) \operatorname{sgn}(s)-\mu(s)]}
\end{aligned}
$$

Because $k>\|\dot{\xi}\|, \quad \eta>0$,So,

$$
\begin{aligned}
\dot{V} & \leq \frac{1}{L} s^{\mathrm{T}}\left(c+p q^{-1} \gamma \operatorname{diag}\left(\dot{\tilde{i}}_{s}^{(p / q)-1}\right)\right)[-\eta \operatorname{sgn}(s)-\mu(s)] \\
& \leq-\frac{1}{L} s^{\mathrm{T}}\left(c+p q^{-1} \gamma \operatorname{diag}\left(\dot{\tilde{\dot{i}}}_{s}^{(p / q)-1}\right)\right)[\eta \operatorname{sgn}(s)+\mu(s)] \\
& \leq-\frac{1}{L} s^{\mathrm{T}}\left(c+p q^{-1} \min _{j=\alpha, \beta}\left(\gamma_{j} \dot{\tilde{i}}_{j}^{(p / q)-1}\right)\right)\left[\eta\|s\|+\mu\|s\|^{2}\right] \\
& \leq 0
\end{aligned}
$$

Where $\operatorname{diag}\left(\dot{\tilde{i}}_{s}^{(p / q)-1}\right)=\left[\begin{array}{cc}\dot{\tilde{i}}_{\alpha}\left({ }^{(p / q)-1}\right) & 0 \\ 0 & \left.\dot{\tilde{i}}_{\beta}(p / q)-1\right)\end{array}\right]$.

Because $1<p / q<2$, and $p, q$ are odd numbers, $(p / q-1)$ is even, namely $\left(\dot{\tilde{i}}_{s}^{(p / q)-1}\right) \geq 0$.

Convergence time calculation:

The time from $s(0) \neq 0$ to $\tilde{i}_{s}\left(t_{s}\right)=0$ is:

$t_{s}=\max _{j=\alpha, \beta}\left[c \ln \left|\tilde{i}_{s}\left(t_{r}\right)\right|+\gamma p(p-q)^{-1} \max _{j=\alpha, \beta}\left(\left|\tilde{i}_{s}\left(t_{r}\right)\right|^{p-q / p}\right)\right]$

$\tilde{i}_{s}$ and $\dot{\tilde{i}}$ will converge to zero in finite time. It can be seen from the equation that the convergence time can be regulated 


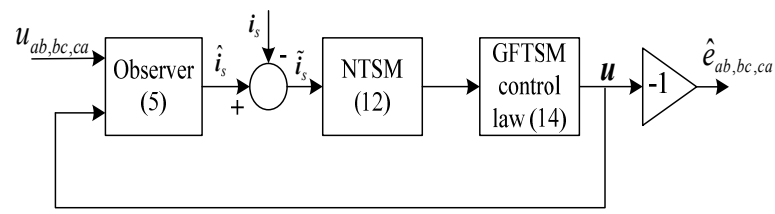

Fig. 4. Structure of the GFTSMO.

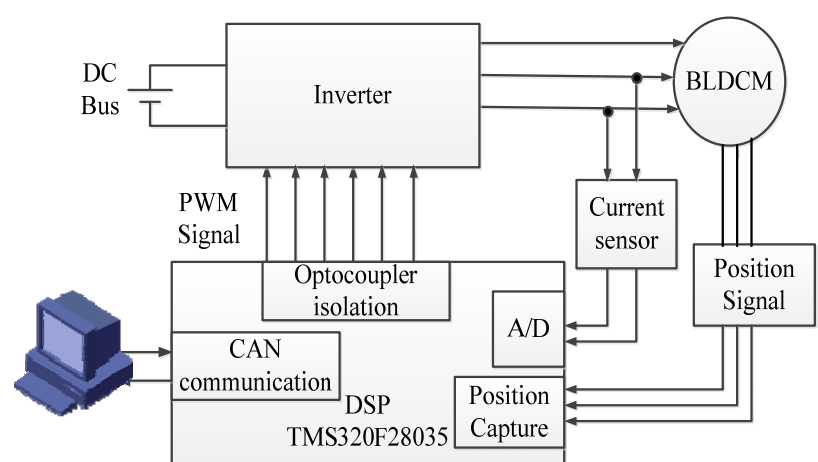

(a)

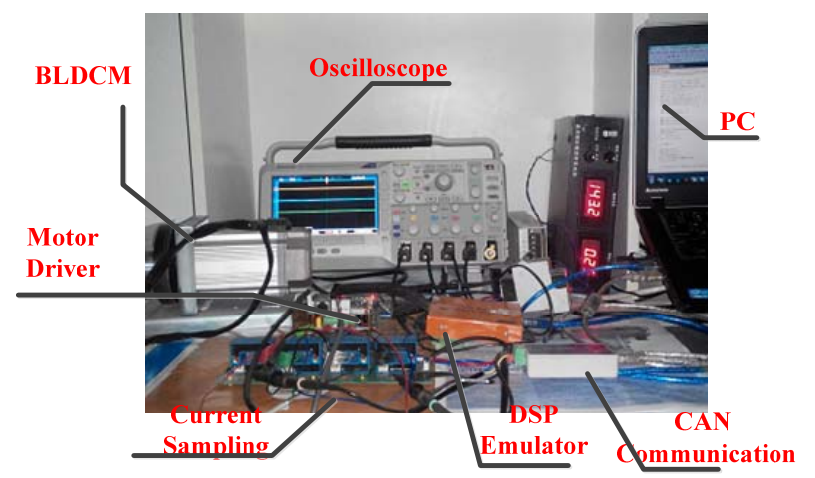

(b)

Fig. 5. Experimental control system. (a) Structure diagram. (b) Experimental setup.

with the parameters $\gamma, p$ and $q$.

The value of the back EMF can be observed by equation (11). The zero crossing point of the line back EMF is the commutation point of the BLDCM. The structure of the GFTSMO is shown in Fig. 4.

\section{EXPERIMENT RESULTS AND ANALYSIS}

An experiment was carried out with the BLDCM control system to validate the feasibility of the proposed method. An experimental platform with a TI control chip TMS320F28035 was built. It consists of a switching logic module, a current sampling module, a power supply module and a BLDCM. The experimental control system structure diagram and real photo are shown in Fig. 5. The BLDCM parameters are as following: the rated power is $3 \mathrm{~kW}$, the rated voltage is $150 \mathrm{~V}$, the rated speed is $3000 \mathrm{r} / \mathrm{min}$, the stator resistance is $0.26 \Omega$, and the stator inductance is $2.5 \mathrm{mH}$. The parameters of the GFTSMO are as following: $p=9, q=7$,

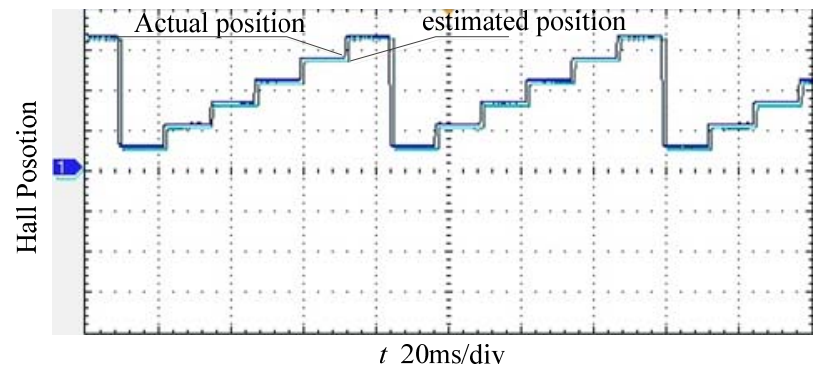

(a)

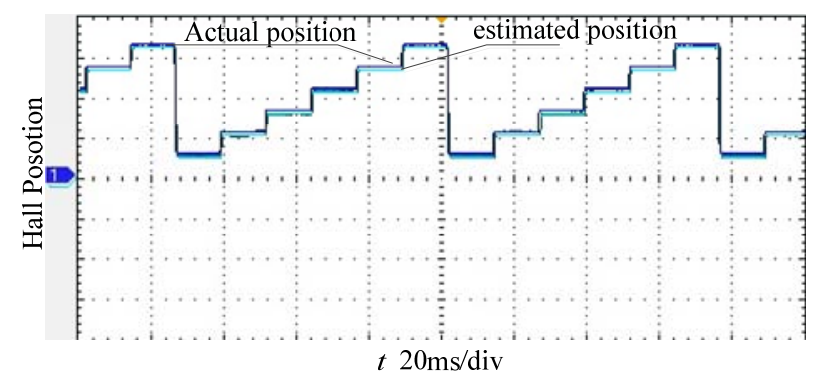

(b)

Fig. 6. Actual and estimated Hall position at speed of 400r/min. (a) Conventional method. (b) The proposed method.

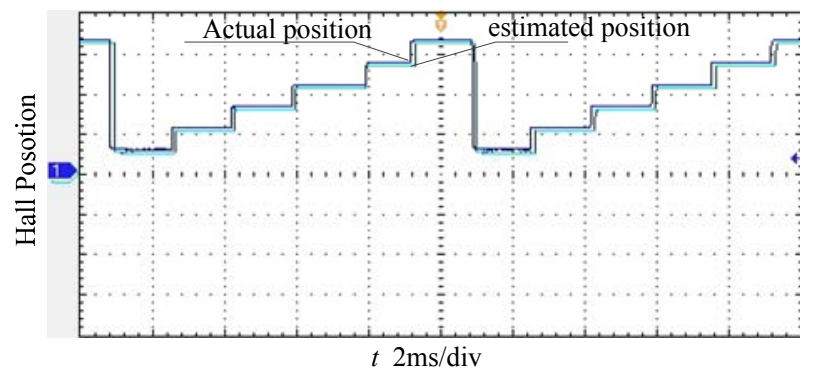

(a)

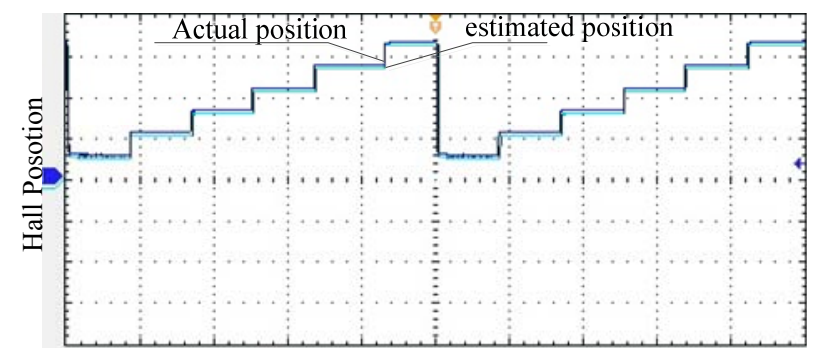

$t 2 \mathrm{~ms} / \mathrm{div}$

(b)

Fig. 7. Actual and estimated Hall position at speed of $3000 \mathrm{r} / \mathrm{min}$. (a) conventional method. (b) The proposed method.

$\sigma=1, \gamma=0.07, k=180, h=2, \zeta=5$, and $\mu=10$.

The rotor position is detected when the motor in the operation mode to verify the performance of the proposed method applied in the position sensorless control of the BLDCM. The estimated position signal is compared with the actual Hall signal. The experimental results are shown in Fig. 6-7 at the speed of $400 \mathrm{r} / \mathrm{min}$ and $3000 \mathrm{r} / \mathrm{min}$, respectively. When the conventional control method is used, there is some deviation between the estimated rotor commutation position 


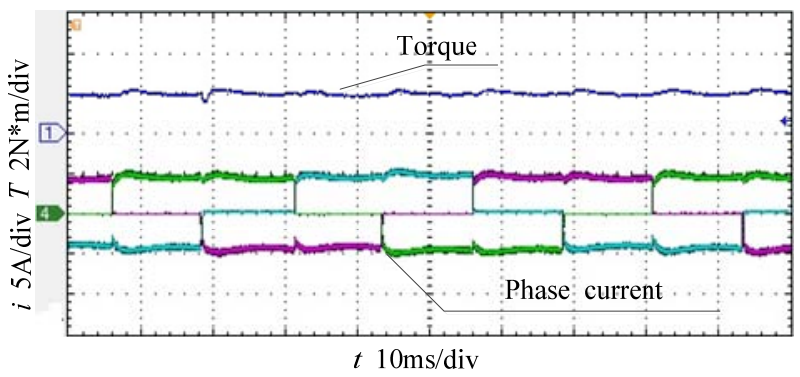

(a)

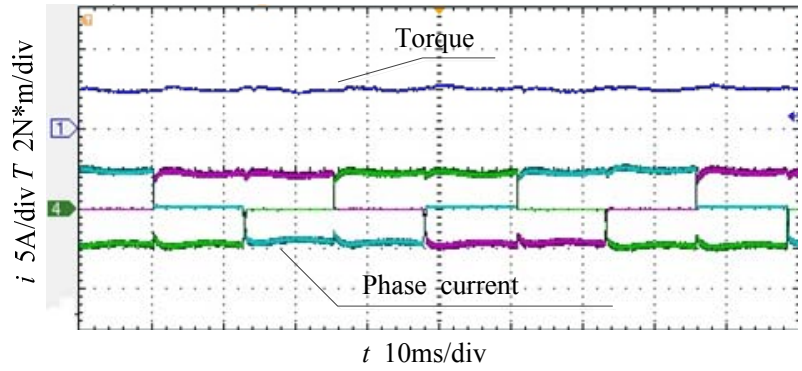

(b)

Fig. 8. Phase current and torque waveform at speed of 200r/min. (a) Conventional method. (b) The proposed method.

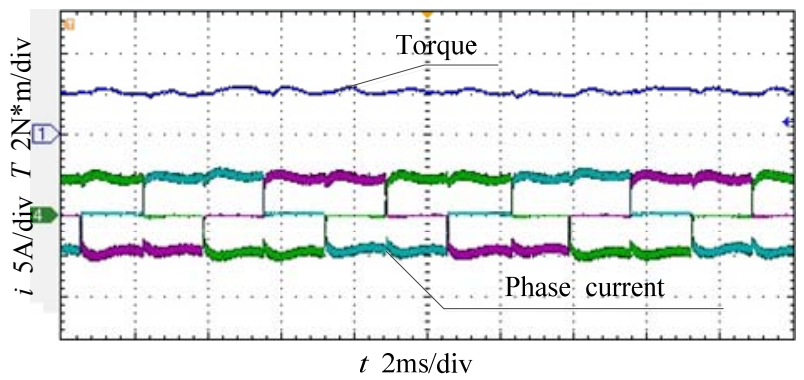

(a)

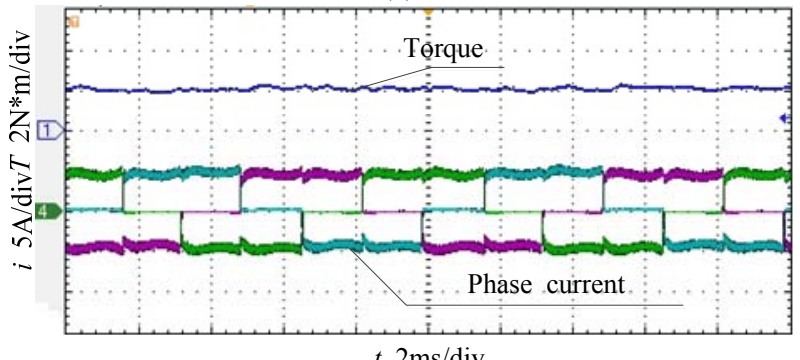

(b)

Fig. 9. Phase current and torque waveform at speed of 3000r/min. (a) Conventional method. (b) The proposed method.

and the actual Hall position. When the proposed control method is adopted, the error decreases significantly. The rotor position can be traced effectively at low or high speed. There is more accuracy in the commutation position estimation of the proposed method when compared with that of the conventional method.

The torque and three phase current waveforms of the motor at the speed of $400 \mathrm{r} / \mathrm{min}, 3000 \mathrm{r} / \mathrm{min}$ are shown in Fig. 8-9, respectively. There is some error between the estimated commutation point and the actual Hall position when both

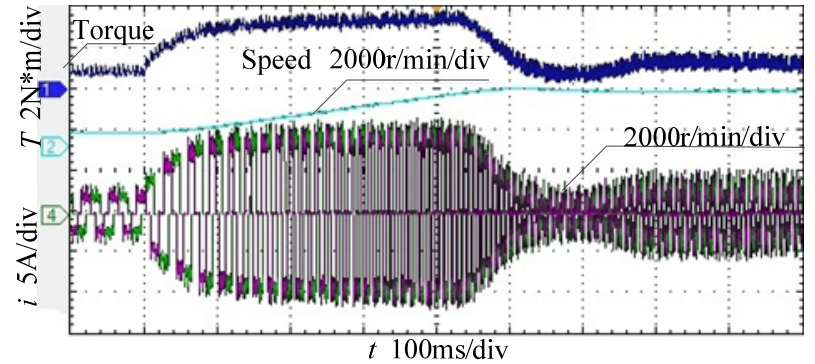

(a)

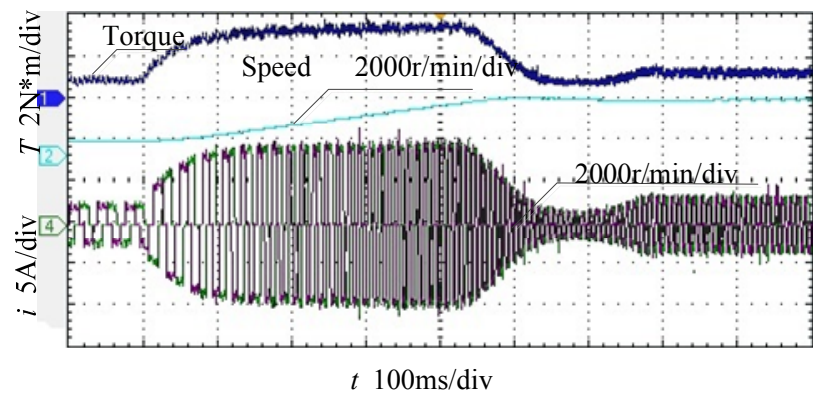

(b)

Fig. 10. The transient process during motor speed changed. (a) Conventional method. (b) The proposed method.

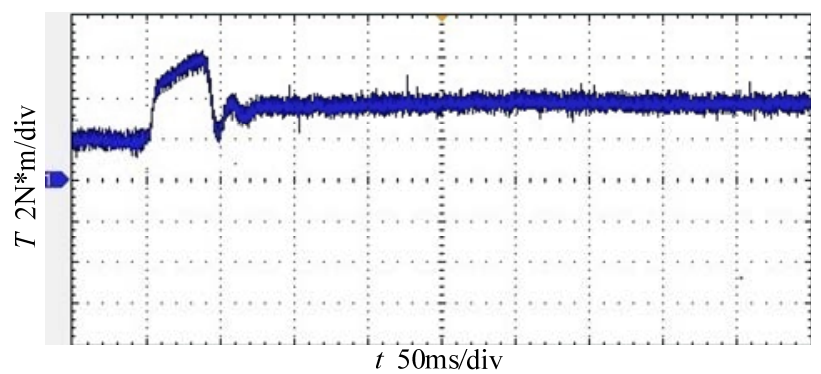

(a)

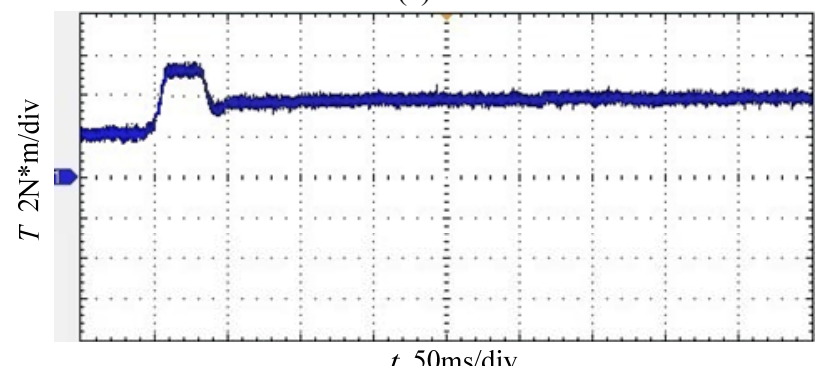

(b)

Fig. 11. Torque waveform when the load changed. (a) Conventional method. (b) The proposed method.

methods are adopted. The error is large when the conventional method is used. The commutation point lags behind the actual position. The current of the three phase is not smooth, and the motor torque ripples are relatively large. However, when the proposed method is used, the position error is smaller when compared to that of the conventional method. Therefore, the motor current is smooth, and the motor torque ripple decreases. The experimental results demonstrate that the proposed method can reduce the motor torque ripple effectively.

Dynamic performance experiments of the BLDCM with 
both methods are carried out. The motor speed changes from $800 \mathrm{r} / \mathrm{min}$ to $3000 \mathrm{r} / \mathrm{min}$. The transient process is shown in Fig. 10. As can be seen, when using the conventional method, the motor reaches the reference speed in $450 \mathrm{~ms}$, the phase current waveform is not good, and the motor torque ripple is large. When the proposed method is used, the time that reaches the reference speed is the same as above. However, the waveforms of the current and the torque waveform are significantly better than those of the conventional method. The error between the estimated commutation point and the actual Hall position is large when the conventional method is used. The commutation point lags behind the actual position. Therefore, with the same load, the current ripple is large. As is shown in Fig. 10(a), the peak of the phase current is larger than that with the proposed method. The good dynamic performance with the proposed method is validated by the experiments. The chattering phenomenon of the motor can be reduced.

The torque response waveform is shown in Fig. 11. The load is changed from $2 \mathrm{~N}^{*} \mathrm{~m}$ to $4 \mathrm{~N}^{*} \mathrm{~m}$ at the speed of $400 \mathrm{r} / \mathrm{min}$. The torque waveform with the conventional control method is shown in Fig. 11 (a). It can be seen that the dynamic time is $50 \mathrm{~ms}$, there is a certain overshoot of the torque and some fluctuation of the torque in the static moment. If the proposed method is adopted, the dynamic time is also about $50 \mathrm{~ms}$. There is less overshoot in the torque and less fluctuation of the torque in the static moment. The experiments show that the convergence speed with the proposed method is faster than that with conventional method.

\section{CONCLUSION}

To achieve a position sensorless control system for the BLDCM of electric vehicles, a global fast terminal SMO is established. To enhance the convergence speed of the SMO and to realize better tracking precision and control performance, the nonsingular terminal SMO and the high order SMO are combined. It makes the whole system have the advantages of global fast convergence. The experiments results showed that the proposed control method can realize commutation control without a position sensor. It has a fast dynamic response and can reduce the chattering phenomenon. The convergence speed is fast when the torque changes. The proposed method has improved robustness and can reduce the torque ripple.

\section{REFERENCES}

[1] S. K. Cheng and S. M. Cui, "A controller of brushless DC motor for electric vehicle," IEEE Trans. Magn., Vol. 41, No. 1, pp. 509-513, Jan. 2005.

[2] J. T. Chen, Z. Q. Zhu, and S. Iwasaki, "A novel hybrid-excited switched-flux brushless AC machine for EV/HEV applications," IEEE Trans. Veh. Technol., Vol. 60, No. 4, pp. 1365-1373, Mar. 2011.

[3] K. T. Chau, C. C. Chan, and C. Liu, "Overview of permanent-magnet brushless drives for electric and hybrid electric vehicles," IEEE Trans. Ind. Electron., Vol. 55, No. 6, pp. 2246-2257, Jun. 2008.

[4] P. Damodharan and K. Vasudevan, "Sensorless brushless DC motor drive based on the zero-crossing detection of back electromotive force (EMF) from the line voltage difference," IEEE Trans. Energy Convers., Vol. 25, No. 3, pp. 661-668, Mar. 2010.

[5] Y. Liu, Z. Q. Zhu, and D. Howe, "Instantaneous torque estimation in sensorless direct-torque-controlled brushless DC motors," IEEE Trans. Ind. Appl., Vol. 42, No. 5, pp. 1275-1283, Oct. 2006.

[6] T. Kim, H. W. Lee, and M. Ehsani, "Position sensorless brushless DC motor/generator drives: Review and future trends," IET Electric Power Appl., Vol. 1, No. 4, pp. 557-564, Jul. 2007.

[7] J. Shao, "An improved microcontroller-based sensorless brushless DC (BLDC) motor drive for automotive applications," IEEE Trans. Ind. Appl., Vol. 42, No. 5, pp. 1216-1221, Oct. 2006.

[8] H. Fakham, M. Djemai, and K. Busawon, "Design and practical implementation of a back-EMF sliding-mode observer for a brushless DC motor." IET Electric Power Applications, Vol. 2, No. 6, pp. 353-361, Nov. 2008.

[9] X. Z. Zhang and Y. N. Wang, "A novel position-sensorless control method for brushless DC motors," Energy Conversion and Management, Vol. 2, No. 6, pp. 1669-1676, Mar. 2011.

[10] C. Lascu and G. Andreescu, "Sliding-mode observer and improved integrator with DC-offset compensation for flux estimation in sensorless-controlled induction motors," IEEE Trans. Ind. Electron., Vol. 53, No. 3, pp. 785-794, Jun. 2006.

[11] J. Yang, S. Li, and X. Yu, "Sliding-mode control for systems with mismatched uncertainties via a disturbance observer," IEEE Trans. Ind. Electron., Vol. 60, No. 1, pp.160-169, Sep. 2013.

[12] H. Kim, J. Son, and J. Lee, "A high-speed sliding-mode observer for the sensorless speed control of a PMSM," IEEE Trans. Ind. Electron., Vol. 58, No. 9, pp. 4069-4077, Dec. 2011.

[13] Y. Lingling, Z. Yun, and C. Zhenfeng, "Bilinear control based on model bias separation for uncertain nonlinear systems," Acta Automatica Sinica, Vol. 36, No. 10, pp. 1432-1442, Oct. 2010.

[14] K. L. Kang, J. M. Kim, K. B. Hwang, and K. H. Kim, "Sensorless control of PMSM in high speed range with iterative sliding mode observer," Applied Power Electronics Conference and Exposition, 2004. APEC'04. Nineteenth Annual IEEE, Vol. 2, 2004.

[15] C. Li and M. Elbuluk, "A robust sliding mode observer for permanent magnet synchronous motor drives," IECON 02, Vol. 2, 2002.

[16] J. Yang, J. Su, S. Li, and X. Yu, "High-order mismatched disturbance compensation for motion control systems via a continuous dynamic sliding-mode approach," IEEE Trans. Ind. Informat., Vol. 10, No. 1, pp. 604-614, Feb. 2014.

[17] X. Yu and Z. Man, "Model reference adaptive control systems with terminal sliding modes," International Journal of Control, Vol. 64, No. 6, pp. 1165-1176, Aug. 1996.

[18] J. Yang, S. Li, J. Su, and X. Yu, "Continuous nonsingular terminal sliding mode control for systems with mismatched disturbances," Automatica, Vol. 49, No. 7, pp. 2287-2291, Jul. 2013.

[19] Y. Feng, X. Yu, and Z. Man, "Non-singular terminal sliding 
mode control of rigid manipulators," Automatica, Vol. 38, No. 12, pp. 2159-2167, Dec. 2002.

[20] X. Yu and M. Zhihong, "Fast terminal sliding-mode control design for nonlinear dynamical systems," IEEE Circuits and Systems Society, Vol.49, No. 2, pp. 261-264, Feb. 2002.

[21] C. Edwards and S. Spurgeon, Sliding Mode Control: Theory and Applications, CRC Press, 1998.

[22] Y. Liu, Z. Q. Zhu, and D. Howe, "Direct torque control of brushless DC drives with reduced torque ripple," IEEE Trans. Ind. Appl., Vol. 41, No. 2, pp. 599-608, Mar. 2005.

[23] S. B. Ozturk, W. C. Alexander, and H. A. Toliyat, "Direct torque control of four-switch brushless DC motor with non-sinusoidal back EMF," IEEE Trans. Power Electron., Vol. 25, No. 2, pp. 263-271, Feb. 2010.

[24] Y. Feng, X. Han, Y. Wang, and X. Yu, "Second-order terminal sliding mode control of uncertain multivariable systems," International Journal of Control, Vol. 80, No. 6, pp. 856-862, Dec. 2007.

[25] Y. Feng, J. Zheng, X. Yu, and N. V. Truong, "Hybrid terminal sliding-mode observer design method for a permanent-magnet synchronous motor control system," IEEE Trans. Ind. Electron., Vol.56, No. 9, pp. 2159-2167, Mar. 2009.

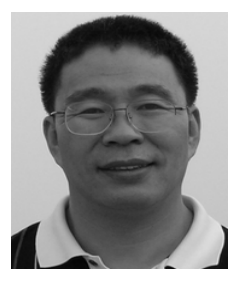

Xiaoyuan Wang was born in Hebei, China, in 1962. He received the B.S. and M.S. degrees in Electrical Engineering from Tianjin University, Tianjin, China, in 1982 and 1985, respectively; and the Ph.D. degree from Shenyang University of Technology, Shenyang, China, in 2006. He is currently a Professor in the School of Electrical Engineering and Automation, Tianjin University. His current research interests include the design of permanent-magnet machines for applications ranging from automotive to renewable energy.

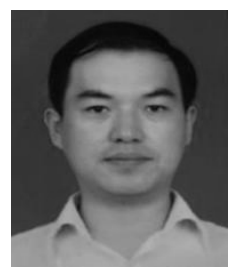

Tao Fu was born in Hebei, China, in 1979. He received the B.S. and M.S. degrees in Electrical Engineering from Tianjin University, Tianjin, China, in 2002 and 2006, respectively; where he is currently working toward the Ph.D. degree in Electrical Engineering in the School of Electrical Engineering and Automation. His current research interests include electrical machines and motor drives.

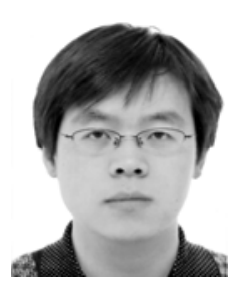

Xiaoguang Wang was born in Hebei, China, in 1984. He received the B.S. degree in Electrical Engineering from Hebei University of Technology, Tianjin, China, in 2006; the M.S. degree in Control Theory and Control Engineering from Xihua University, Chengdu, China, in 2010; and the Ph.D. degree in Electrical Engineering from Tianjin University, Tianjin, China, in 2015. He is currently a Lecturer in the School of Electrical and Electronic Engineering, Hubei University of Technology. His current research interests include electrical machines and motor drives. 